\title{
A Graph Operation and Its Applications in Generating Orderenergetic and Equienergetic Graphs
}

\author{
Subin P. Joseph \\ Department of Mathematics, Government Engineering College, Wayanad, \\ Kerala, India \\ subinpj@gecwyd.ac.in
}

(Received September 17, 2021)

\begin{abstract}
A general graph operation is defined and some of its applications are given in this paper. The adjacency spectrum of any graph generated by this operation is given. A method for generating integral graphs using this operation is discussed. Corresponding to any given graph, we can generate an infinite sequence of pair of equienergetic non-cospectral graphs using this graph operation. Given an orderenergetic graph, it is shown that we can construct two different sequences of orderenergetic graphs. A condition for generating orderenergetic graphs from non-orderenergetic graphs are also derived. This method of constructing connected orderenergetic graphs solves one of the open problem stated in the paper by Akbari et al.(2020).
\end{abstract}

\section{Introduction}

Consider a graph $G=(V, E)$, where $V$ is the vertex set and $E$ is the edge set. The order of this graph is $n=|V|$. Let $A_{G}$ denotes the adjacency matrix of the graph, which is a symmetric matrix with binary entries. The ordinary energy of a graph $G$ is defined to be the sum of the absolute value of the eigenvalues of the adjacency matrix $A_{G}$, which is denoted by $\mathcal{E}_{G}[9]$. Let $\lambda_{1}, \lambda_{2}, \cdots, \lambda_{n}$ are the eigenvalues of $A$, then

$$
\mathcal{E}_{G}=\sum_{i=1}^{n}\left|\lambda_{i}\right|
$$

Graphs can be classified according to their energies. An orderenergetic graph is defined to be graph $G$ with $\mathcal{E}_{G}=n$ [1]. If $\mathcal{E}_{G}<n$, then such a graph is called hypoenergetic 
graph [10] and if $\mathcal{E}_{G}>2 n-2$, such graphs are called hyperenergetic graph [8]. The borderenergetic graphs are having $\mathcal{E}_{G}=2 n-2$ [5]. Equienergetic graphs are those graphs with same order having equal energy [13]. If these are having same spectrum then they are called cospectral graphs, otherwise non-cospectral. Several studies and applications of these different types of graphs can found in $[3,4,7,10,11]$ and references therein, except orderenergetic graphs

Orderenergetic graphs are recently defined in [1] and hence related studies are in the beginning stage in the literature. In [1] the authors have shown that the elements in the family of complete tripartite graphs $K_{p, p, 6 p}$ are orderenergetic graphs where $p$ is any positive integer. In addition to this, all the balanced complete bipartite graphs $K_{p, p}$ are also orderenergetic graphs. These are the only known classes of orderenergetic graphs. In [1] it is also shown that the connected graph obtained by taking the direct product of any two orderenergetic graphs with orders $m$ and $n$, one of which is a non-bipartite graph, will be also a connected orderenergetic graph with order $m n$. They have shown that there are only nine orderenergetic graphs up to order ten [1], using computer search. They stated an open problem regarding construction of new orderenergetic graphs. A new method for generating orderenergetic graphs and equienergetic graphs are presented in this paper.

We define a general graph operation in the next section which produces new graphs from a given graph. The spectrum of these new graphs can be computed from the spectrum of the given graph and this is given in third section. As an application of this graph operation we can construct several infinite sequences of integral graphs, which are graphs with all integer eigenvalues. This operation is also used to find out several pairs of equienergetic non-spectral graphs in two different ways. It is shown that starting with any given graph we can generate an infinite sequence of pair of equienergetic non-cospectral graphs. Another important application of the proposed graph operation is in the generation of several sequences of orderenergetic graphs. It is also shown that we can generate orderenergetic graphs even from non-orderenergetic graphs by means of the this general graph operation. 


\section{A general graph operation}

Let $G$ be a graph with order $n$ and number of edges $m$. Take $p$ copies of $G$, denoted by $G_{i}$, for $1 \leq i \leq p$, and take $n q$ number of isolated vertices for some positive integer $q$. Name the $n$ vertices of $G_{i}$ as $V_{i}=\left\{v_{i 1}, v_{i 2}, \cdots, v_{i n}\right\}$, for $1 \leq i \leq p$. Let the isolated vertices be named as $\left\{u_{j k}\right\}$, where $1 \leq j \leq q$ and $1 \leq k \leq n$. Then construct a new graph $H$ from the graphs $\left\{G_{i}\right\}$ and the isolated vertices $\left\{u_{j k}\right\}$ as follows.

1. The vertex set of $H$ is the union of the vertices in $G_{i}$ and the isolated vertices $\left\{u_{j k}\right\}$.

2. The edge set of $H$ contains all the edges in $G_{i}$ 's . In addition to these edges, the following two types edges will be added in the edge set of $H$. If $v_{i j}$ is adjacent to $v_{i k}$ in $G_{i}$ for $1 \leq i \leq p$, then

- add the edges $\left\{v_{i j}, v_{l k}\right\}$ and $\left\{v_{i k}, v_{l j}\right\}$ for all $1 \leq l \leq n$ and $l \neq i$, i.e., add edges from $v_{i j}$ to every $k^{\text {th }}$ vertex in $G_{l}$ for $l \neq i$ and from $v_{i k}$ to every $j^{\text {th }}$ vertex in $G_{l}$ for $l \neq i$.

- add the edges $\left\{v_{i j}, u_{l k}\right\}$ and $\left\{v_{i k}, u_{l j}\right\}$ for all $1 \leq l \leq q$.

The total number of vertices in the derived graph $H$ will be $n(p+q)$ and the total number of edges will be $m p(p+2 q)$. The above graph construction is illustrated using two examples. The graph $H$ obtained from the complete graph on two vertices $K_{2}$ with $p=2$ and $q=3$ is shown as the first graph given in figure 1. For the second example, $H$ is constructed from the path of length 3 and taking $p=q=2$ and it is the second graph given in figure 1.
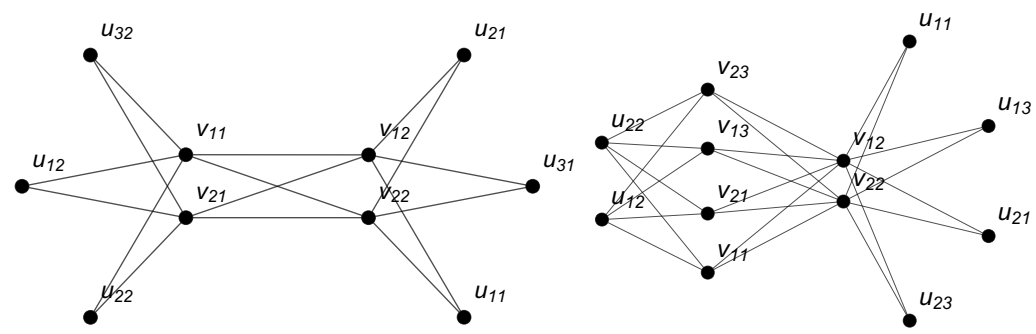

Figure 1. Graphs constructed using the graph operation defined in section 2. The first graph is obtained from two copies of $K_{2}$ and 6 isolated vertices. Second graph is obtained from two copies of path of length two $\left(P_{3}\right)$, and 6 isolated vertices. 


\section{Spectrum of the graph constructed}

The main focus in this paper is to study the spectrum of the graphs constructed using the operation defined above. Spectrum of any graph is needed to find the energy of a graph. Since the graph $H$ is derived from a graph $G$, it will be better to derive the spectrum of it in terms of the spectrum of $G$. For finding the spectrum of a graph we need to represent the graph using its adjacency matrix. It is not hard to see that the adjacency matrix of the new graph $H$ derived from the defined operations is given by the following Kronecker product

$$
A_{H}=J_{p}^{q} \otimes A_{G}
$$

where $A_{G}$ is the adjacency matrix of the graph $G$. Here, $J_{p}^{q}$ is a square matrix of order $p+q$, whose block matrix representation is given by

$$
J_{p}^{q}=\left(\begin{array}{cc}
J_{p} & J_{p q} \\
J_{q p} & \mathbf{0}
\end{array}\right)
$$

where $J_{p q}$ represents the all one matrix of order $p \times q, J_{p}$ represents the all one square matrix of order $p \times p$ and $\mathbf{0}$ represents a zero matrix.

Before giving the statement of the main theorem, we need to state some known results from theory of matrices $[6,12]$.

Lemma 1. If $A$ and $D$ are square matrices(need not be same order) and $B$ and $C$ are matrices with compatible orders, then the determinant of the following block matrix is given by

$$
\operatorname{Det}\left(\begin{array}{ll}
A & B \\
C & D
\end{array}\right)=\operatorname{Det}(D) \operatorname{Det}\left(A-B D^{-1} C\right)
$$

provided $D^{-1}$ exists.

Lemma 2. Let $A$ is an $r^{\text {th }}$ order square matrix with eigenvalues $\left\{\alpha_{i}\right\}, 1 \leq i \leq r$ and $B$ is an $s^{\text {th }}$ order square matrix with eigenvalues $\left\{\beta_{i}\right\}, 1 \leq i \leq s$, then the eigenvalues of the square matrix $A \otimes B$ of order rs is given by all possible products $\left\{\alpha_{i} \beta_{j}\right\}$, for $1 \leq i \leq r$ and $1 \leq j \leq s$, which is rs in number.

Lemma 3. If $B=\left[b_{i j}\right]$ is a $q^{\text {th }}$ order square matrix, then

$$
J_{p q} B J_{q p}=\left(\sum_{i=1}^{q} \sum_{j=1}^{q} b_{i j}\right) J_{p}
$$

Also,

$$
\operatorname{Det}\left(\omega I_{p}-J_{p}\right)=\omega^{n-1}(\omega-p)
$$


Now, we state the main theorem in this paper.

Theorem 1. If $G$ is a graph of order $n$ whose non-zero eigenvalues are given by $\left\{\lambda_{i}\right\}, 1 \leq$ $i \leq r$, for some $r \leq n$, then the non-zero eigenvalues of the graph $H$ are given by

$$
\left\{\frac{1}{2}(p \pm \sqrt{p(p+4 q)}) \lambda_{i}\right\}
$$

for $1 \leq i \leq r$, which are $2 r$ in number. Also, zero is an eigenvalue of $H$ with multiplicity $n(p+q)-2 r$.

Proof. Let $A_{G}$ and $A_{H}$ are the adjacency matrices of the graphs $G$ and $H$ respectively. It is required to find out the eigenvalues of $A_{H}$. First of all we compute the characteristic polynomial of the matrix $J_{p}^{q}$ and then apply the formula (2) and Lemma 2. Using equation (3), lemma 1 and lemma 3, the characteristic polynomial of the matrix $J_{p}^{q}$ is given by

$$
\begin{aligned}
f_{J_{p}^{q}}(\lambda) & =\operatorname{Det}\left(\lambda I_{p+q}-J_{p}^{q}\right)=\operatorname{Det}\left(\begin{array}{cc}
\lambda I_{p}-J_{p} & J_{p q} \\
J_{q p} & \lambda I_{q}
\end{array}\right) \\
& =\operatorname{Det}\left(\lambda I_{q}\right) \operatorname{Det}\left(\left(\lambda I_{p}-J_{p}\right)-J_{p q}\left(\lambda I_{q}\right)^{-1} J_{q p}\right) \\
& =\lambda^{q} \operatorname{Det}\left(\left(\lambda I_{p}-J_{p}\right)-\frac{1}{\lambda} J_{p q} I_{q} J_{q p}\right) \\
& =\lambda^{q} \operatorname{Det}\left(\left(\lambda I_{p}-J_{p}\right)-\frac{q}{\lambda} J_{p}\right)=\lambda^{q} \operatorname{Det}\left(\lambda I_{p}-\frac{\lambda+q}{\lambda} J_{p}\right) \\
& =\lambda^{q}\left(\frac{\lambda+q}{\lambda}\right)^{p} \operatorname{Det}\left(\frac{\lambda^{2}}{\lambda+q} I_{p}-J_{p}\right)=\lambda^{q}\left(\frac{\lambda+q}{\lambda}\right)^{p}\left(\frac{\lambda^{2}}{\lambda+q}\right)^{p-1}\left(\frac{\lambda^{2}}{\lambda+q}-p\right) \\
& =\lambda^{p+q-2}\left(\lambda^{2}-\lambda p-p q\right)
\end{aligned}
$$

So, the non-zero eigenvalues of the matrix $J_{p}^{q}$ are $\frac{1}{2}(p \pm \sqrt{p(p+4 q)})$ and the eigenvalue zero has multiplicity $p+q-2$. Then, from the equation (2) and the lemma 2 the theorem follows.

\section{Applications of the new graph operation}

The graph $H$ obtained from the operations defined in this paper from a given graph $G$ is denoted by

$$
H=G_{(p)}^{(q)}
$$

and the graph $G$ is called the seed graph. The adjacency matrix of this graph $H$ is given by equation (2).

We discuss several applications of the graph operation defined and its spectrum in this section. Our next result characterize some of the graphs $H$ which are integral graphs. This gives an efficient way for generating integral graphs from known integral graphs. 
Theorem 2. Let $G$ is an integral graph of order $n$. If $p$ is not a perfect square number, then the graph $H$ is an integral graph when $q=\operatorname{pr}(r+1)$, for any non-negative integer $r$. If $p$ is a perfect square, then also the graph $H$ is an integral graphs provided $q=(s+r) r$ for any non-negative integer $r$, where $p=s^{2}$.

Proof. Let $G$ is an integral graph. Then from theorem 1, $H$ is also an integral graph if and only if $\frac{1}{2}(p \pm \sqrt{p(p+4 q)})$ are integers. This will happen only when $p(p+4 q)$ is a perfect square. We will consider two cases separately. In the first case let $p$ is not a perfect square. Then, to make $p(p+4 q)$ a perfect square, let $q=p f(r)$ where $f(r)$ is non-negative integer valued function. Then

$$
p(p+4 q)=p^{2}(1+4 f(r))
$$

The general integer valued function making this a perfect square is $f(r)=r(r+1)$, so that

$$
p(p+4 q)=p^{2}(2 r+1)^{2}
$$

So the required value of $q=\operatorname{pr}(r+1)$. Then the eigenvalues of $J_{p}^{p r(r+1)}$ becomes $p(r+1)$ and $-p r$, for any non-negative integer $r$. So, from theorem 1 it follows that the eigenvalues of $H$ are integers and so $H$ is an integral graph if $G$ is an integral graph.

In the second case, we assume that $p$ is a perfect square and let $p=s^{2}$ for some positive integer $s$. Then to make $p(p+4 q)=s^{2}\left(s^{2}+4 q\right)$ a perfect square we need to put $q=r(s+r)$. Then $p(p+4 q)=s^{2}(s+2 r)^{2}$. This is true for any non-negative integer $r$. Then the eigenvalues of $J_{p}^{q}=J_{s^{2}}^{(s+r) r}$ becomes $s^{2}+s r$ and $-s r$, for any non-negative integer $r$ and $p=s^{2}$. Hence, from lemma 2 and equation (2) it follows that the eigenvalues of $H$ are integers and $H$ is an integral graph, provided $G$ is an integral graph.

The next theorem gives a general method for generating orderenergetic graphs from a given graph.

Theorem 3. Let $G$ is any graph on $n$ vertices with integral energy $\mathcal{E}_{G}$. If $r$ is a nonnegative integer satisfying

$$
\mathcal{E}_{G}=\frac{n\left(r^{2}+r+1\right)}{2 r+1},
$$

then the graph $H=G_{(p)}^{(q)}$ with $q=\operatorname{pr}(r+1)$ is always an orderenergetic graph for any arbitrary positive integer $p$. 
Proof. Let $r$ is a non-negative integer satisfying equation (11) where $\mathcal{E}_{G}$ is the integral energy of the graph $G$. Let $A_{H}$ be the adjacency matrix of the graph $H$ with $q=\operatorname{pr}(r+1)$ . Then it follows that $\mathcal{E}_{H}=p(2 r+1) \mathcal{E}_{G}$, using theorem 2. Now, using equation (11), we have

$$
\mathcal{E}_{H}=p(2 r+1) \mathcal{E}_{G}=n p\left(r^{2}+r+1\right)=n(p+p(r(r+1))=n(p+q),
$$

where $n(p+q)$ is the order of the graph $H$. So the graph $H$ is an orderenergetic graph.

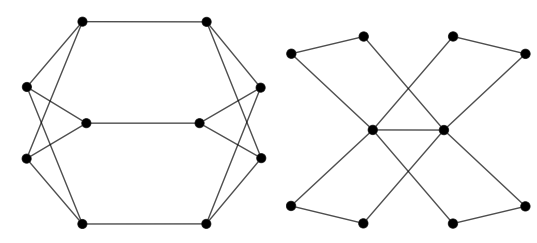

Figure 2. Two non-orderenergetic graphs used for generating orderenergetic graphs (see theorem 3 and note 2).

Corollary 1. If $G$ is an orderenergetic graph on $n$ vertices, then the sequence of graphs $G_{(p)}^{(0)}$ are also orderenergetic graphs with order pn for any positive integer $p$.

Proof. The result is clear from theorem 3 by letting $r=0$ and noting that $\mathcal{E}_{G}=n$.

Corollary 2. If $G$ is an orderenergetic graph on $n$ vertices, then the sequence of graphs $G_{(p)}^{(2 p)}$ are also orderenergetic graphs with order $3 p n$ for any positive integer $p$.

Proof. The result follows from theorem 3 by letting $r=1$ and noting that $\mathcal{E}_{G}=n$.

Note 2. It is clear from above corollaries that for any orderenergetic graph we can generate two different sequences of orderenergetic graphs as a consequence of theorem 3. Then the natural question that arise is that whether we can generate orderenergetic graphs from some non-orderenergetic graphs using this theorem 3 or not. Indeed it is possible, which is illustrated using two examples here. Consider the two graphs with order 10 given in figure 2. These graphs are equienergetic graphs with energy 14. Then from theorem 3 we obtain the value of $r=2$ and it is concluded that the sequence of graphs $G_{(p)}^{(p r(r+1))}=G_{(p)}^{(6 p)}$ for each of the seed graphs given in figure 2 are all orderenergetic graphs. So, we can generate orderenergetic graphs from non-orderenergetic graphs using 
theorem 3 .

The next two corollaries gives two different ways to generate equienergetic graphs.

Corollary 3. Let $G$ and $\hat{G}$ be a pair of equienergetic graphs, then the graphs $G_{(p)}^{(q)}$ and $\hat{G}_{(p)}^{(q)}$ are also pair of equienergetic graphs for any positive integer $p$.

Proof. The result follows from theorem 1 and equation (7).

Our next corollary gives an efficient way for constructing a sequence of pair of equienergetic graphs which are non-cospectral in general, from a given arbitrary graph. Also, if the given graph is integral we are getting an infinite sequence of pair of equienergetic non-cospectral integral graphs.

Corollary 4. Let $G$ be any graph, then the pair of graphs $G_{(3 p)}^{(0)}$ and $G_{(p)}^{(2 p)}$ are noncospectral equienergetic graphs of same order, for any positive integer $p$. Moreover, if the graph $G$ is an integral graph, then all the pair of equienergetic graphs are also integral graphs.

Proof. Let the non-zero eigenvalues of $G$ are $\left\{\lambda_{i}\right\}, 1 \leq i \leq r$, for some $r \leq n$ and the energy is $\mathcal{E}_{G}$. Then from theorem 1 it follows that the non-zero eigenvalues of $G_{(3 p)}^{(0)}$ and $G_{(p)}^{(2 p)}$ are $\left\{3 p \lambda_{i}\right\}, 1 \leq i \leq r$ and $\left\{2 p \lambda_{i},-p \lambda_{i}\right\}, 1 \leq i \leq r$ respectively. Hence the graphs $G_{(3 p)}^{(0)}$ and $G_{(p)}^{(2 p)}$ are clearly non-cospectral and equienergetic with energy $3 p \mathcal{E}_{G}$. Finally, it follows that if the graph $G$ is an integral graph, then all these pair of graphs are integral graphs.

\section{Prime orderenergetic graphs}

In [1] it is also shown that the elements in the family of complete tripartite graphs $K_{p, p, 6 p}$ are orderenergetic graphs where $p$ is any positive integer. In addition to this, all the balanced complete bipartite graphs $K_{p, p}$ are also orderenergetic graphs. These are the known classes of orderenergetic graphs of order $8 p$ and $2 p$ respectively. In [1] it is also shown that the connected graph obtained by taking the direct product of any two orederenergetic graphs with orders $m$ and $n$, one of which is a non-bipartite graph, will be also a connected orderenergetic graph with order $m n$. They also found that there are only nine orderenergetic graphs up to order ten [1]. These graphs are tabulated below. 
Table 1. Table of orderenergetic graphs up to order 10

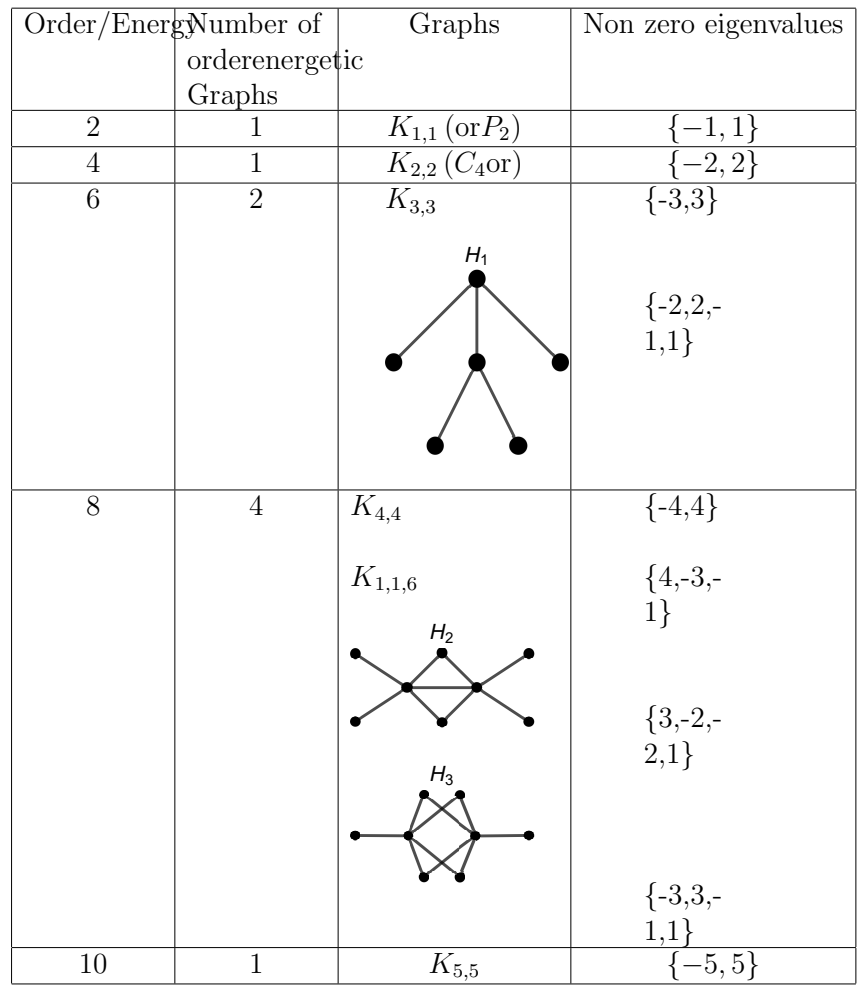

In this section we characterize certain family of ordenergetic graphs. A graph is said to be prime orderenergetic graph if it is orderenergetic and it is not of the form $G_{(p)}^{(0)}$ with $p>1$ or $G_{(p)}^{(2 p)}$ with $p \geq 1$ for any orderenergetic graph $G$.

Theorem 4. The graphs $K_{1,1}, K_{1,1,6}, H_{2}$ and $H_{3}$ given in table 1 are prime orderenergetic graphs and the graphs $K_{2,2}, K_{3,3}$ and $H_{1}$ are not prime orderenergetic graphs.

Proof: First of all note that, if $G$ is of order $n$, then $G_{(p)}^{(0)}$ and $G_{(p)}^{(2 p)}$ are with orders $n p$ and $3 n p$ respectively. Clearly $K_{1,1}$ is the smallest orderenergetic graph, and so it is clearly a prime orderenergetic graph. Since the order of $K_{1,1,6}$ is 8 , it is not prime orderenergetic only if it is of the form $G_{(2)}^{(0)}$ for a fourth order orderenergetic graph $G$ or it is of the form $G_{(4)}^{(0)}$ for a second order orderenergetic graph $G$. But, the only fourth order orderenergetic graph is $G=K_{2,2}$ and the corresponding graph $G_{(2)}^{(0)}$ is the graph $K_{4,4}$. So, the first case 
is not possible. In the second case, for the second order orderenergetic graph $G=K_{1,1}$, the graph $G_{(4)}^{(0)}$ is also $K_{4,4}$. So it follows that $K_{1,1,6}$ is a prime orderenergetic graph.

Similar to the proof of the graph $K_{1,1,6}$, we can show that the orderenergetic graphs $H_{2}$ and $H_{3}$ of order 8 in table 1 are also prime orderenergetic.

Now, consider the orderenergetic tree $H_{1}$ of order 6 in the table. Clearly it is not a prime orderenergetic graph as $H_{1}=G_{(1)}^{(2)}$, where $G=K_{1,1}$. It is also easy to see that $K_{2,2}=G_{(2)}^{(0)}$ and $K_{3,3}=G_{(3)}^{(0)}$ where $G=K_{1,1}$. So, they are also not prime orderenergetic graphs.

If $G$ is a prime orderenergetic graph, then from corollary 1 and corollary 2, there are two sequence of orderenergetic graphs $G_{(p)}^{(0)}$ and $G_{(p)}^{(2 p)}$ generated by $G$. Now, we characterize the first sequence of orderenergetic graphs generated by the prime orderenergetic graph $K_{1,1}$. Before that we prove the following result for an orderenergetic bipartite graph.

Theorem 5. If $G$ is an orderenergetic bipartite graph of order $n$, then all the graphs $H_{m}=G_{(m)}^{(0)}$ are also orderenergetic bipartite graphs with order $m n$.

Proof: Let $G=(V, E)$ be a bipartite graph, where $V=V_{1} \cup V_{2}$ with $\left|V_{1}\right|=r,\left|V_{2}\right|=s$ and $|V|=n=r+s$. Then the adjacency matrix $A_{G}$ of this graph can be represented as a block matrix given by

$$
A_{G}=\left[\begin{array}{cc}
\mathbf{0} & B \\
B^{T} & \mathbf{0}^{\prime}
\end{array}\right] .
$$

Here, $\mathbf{0}$ is the zero square matrix with order $r, B$ is the binary matrix of order $r \times s$, $B^{T}$ represents the transpose of the matrix $B$ and $\mathbf{0}^{\prime}$ is the zero square matrix of order $s$. Since $J_{m}$ and $A_{G}$ are square matrices, we have

$$
J_{m} \otimes A_{G}=P\left(A_{G} \otimes J_{m}\right) P^{T},
$$

where $P$ is some permutation matrix $[6,14]$. So, from equation $(2)$, it follows that the adjacency matrix of the graph $H_{m}$ is

$$
\begin{aligned}
A_{H_{m}} & =P\left(A_{G} \otimes J_{m}\right) P^{T} \\
& =P\left(\left[\begin{array}{cc}
\mathbf{0} & B \\
B^{T} & \mathbf{0}^{\prime}
\end{array}\right] \otimes J_{m}\right) P^{T} \\
& =P\left[\begin{array}{cc}
\mathcal{O} & B \otimes J_{m} \\
B^{T} \otimes J_{m} & \mathcal{O}^{\prime}
\end{array}\right] P^{T} \\
& =P \mathcal{A}_{m} P^{T}
\end{aligned}
$$

where $\mathcal{O}$ and $\mathcal{O}^{\prime}$ are zero square matrices of order $\mathrm{rm}$ and $\mathrm{sm}$ respectively and

$$
\mathcal{A}_{m}=\left[\begin{array}{cc}
\mathcal{O} & B \otimes J_{m} \\
B^{T} \otimes J_{m} & \mathcal{O}^{\prime}
\end{array}\right]
$$


is a binary matrix with order $m n$. It is clear from equation (16) and the fact that transposition is distributive over Kronecker product, the graph $\mathcal{G}_{m}$ with adjacency matrix $\mathcal{A}_{m}$ is a bipartite graph of order $m n$, with $r m$ number of vertices in one set and $s m$ number of vertices in the second set. It follows that $H_{m}$ is also a bipartite graph, since the graphs $H_{m}$ and $\mathcal{G}_{m}$ are isomorphic, as evident from equation (15). Hence, the sequence of graphs $H_{m}=G_{(m)}^{(0)}$ are orderenergetic bipartite graphs, if the graph $G$ is orderenergetic bipartite graph.

Now we can characterize the balanced complete bipartite graphs in the following theorem.

Theorem 6. The sequence of orderenergetic graphs $G_{(m)}^{(0)}$ generated by the prime orderenergetic graph $G=K_{1,1}$ is the sequence of all orderenergetic balanced complete bipartite graphs $K_{m, m}$.

Proof: It follows from corollary 1 that the graphs $G_{(m)}^{(0)}$ are all orderenergetic balanced bipartite graphs of order $2 m$. It is enough to show that they are complete bipartite graphs also. From equation (16), it follows that the adjacency matrix of the graph $G_{(m)}^{(0)}$ is

$$
\left[\begin{array}{ll}
\mathcal{O} & J_{m} \\
J_{m} & \mathcal{O}
\end{array}\right]
$$

where $\mathcal{O}$ is the zero matrix of order $m$. Since $J_{m}$ is the all one matrix, from equation (17) it follows that the orderenergetic graphs $G_{(m)}^{(0)}$ are the balanced complete bipartite graphs of order $2 m$ and hence $G_{(m)}^{(0)}=K_{m, m}$.

The next theorem, which characterize the orderenergetic complete tripartite graphs $K_{p, p, 6 p}$, can also be proved in the same way.

Theorem 7. The sequence of orderenergetic graphs $G_{(m)}^{(0)}$ generated by the prime orderenergetic graph $G=K_{1,1,6}$ is the sequence of all orderenergetic complete tripartite graphs $K_{m, m, 6 m}$.

In the next theorem we show that orderenergetic graphs of certain orders are always prime orderenergetic graphs, except for balanced complete bipartite graphs.

Theorem 8. Any orderenergetic graph of order $2 p$, apart from balanced complete bipartite graph $K_{p, p}$, is a prime orderenergetic graph for any prime number $p>3$.

Proof: Suppose order of the orderenergetic graph $\hat{G}$ is $2 p$ and it is not a balanced complete bipartite graph, where $p$ is a prime number. Since the order of the graph $G_{(m)}^{(2 m)}$ 
is always a multiple of $3, \hat{G}$ will not be appear in this sequence for any orderenergetic graph $G$, since $p>3$. Suppose $\hat{G}=G_{(m)}^{(0)}$ for some orderenergetic graph $G$. Then $2 p=m n$, where $n$ is the order of $G$. Then the possibilities are either $m=2$ and $n=p$ or $m=p$ and $n=2$. The first case is impossible as there are no orderenergetic graph of odd order [2]. In the second case the Kronecker product will lead to the balanced complete bipartite graph $K_{p, p}$. So, the original graph $\hat{G}$ cannot be expressed in the form $G_{(m)}^{(0)}$ with $m>1$

or $G_{(m)}^{(2 m)}$ with $m \geq 1$ for any orderenergetic graph $G$. Hence, given any orderenergetic graph $\hat{G}$ of order $2 p$, apart from balanced complete bipartite graph $K_{p, p}$, it is always prime orderenergetic graph for any prime number $p>3$.

Note 3. When $p=3$, the only orderenergetic graph of order $2 p=6$ is the tree $H_{1}$ (excluding the balanced complete bipartite graph) given in table 1 and it is already shown this is not a prime orderenergetic graph. As discussed in the earlier parts of this paper, it is clear that there exist no orderenergetic graphs with order $2 p$, for the primes $p=2$ or $p=5$, other than balanced complete bipartite graphs.

Since the problem of finding orderenergetic graphs with higher orders is a difficult task, the question that naturally arise is that whether there exist orderenergetic graphs of order $2 p$ for other prime numbers or not. If there exist such graphs, then they are all prime orderenergetic graphs by the above theorem. We have got a positive result for the next prime $p=7$ by a computer search. One of the prime orderenergetic graphs of order 14 is given in figure 3.

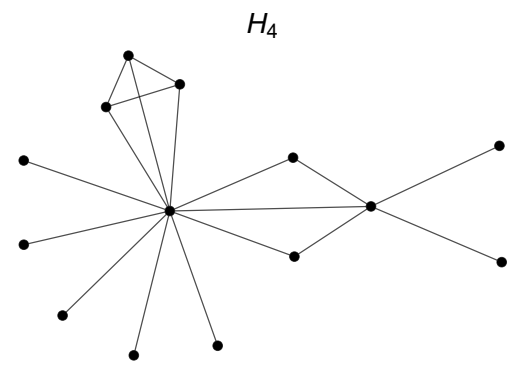

Figure 3. Prime orederenergetic graph of order 14

From the various result discussed in this paper, we can conclude the following corollary which gives the existence of orderenergetic graphs for various orders. 
Corollary 5. There exist orderenergetic graphs of order $6 m, 8 m$ and $14 m$ for any positive integer $m$, other than balanced complete bipartite graphs.

\section{References}

[1] S. Akbari, M. Ghahremani, I. Gutman, F. Koorepazan-Moftakhar, Orderenergetic graphs, MATCH Commun. Math. Comput. Chem. 84 (2020) 325-334.

[2] R. B. Bapat, S. Pati, Energy of a graph is never an odd integer, Bull. Kerala Math. Assoc. 1 (2004) 129-132.

[3] B. Deng, X. Li, I. Gutman, More on borderenergetic graphs, Lin. Algebra Appl. 497 (2016) 199-208.

[4] B. Furtula, I. Gutman, Borderenergetic graphs of order 12, Iran. J. Math. Chem. 8 (2017) 339-344.

[5] S. Gong, X. Li, G. Xu, I. Gutman, B. Furtula, Borderenergetic graphs, MATCH Commun. Math. Comput. Chem. 74 (2015) 321-332.

[6] A. Graham, Kronecker Products and Matrix Calculus with Applications, Wiley, New York, 1981.

[7] I. Gutman, Hyperenergetic and hypoenergetic graphs, in: D. Cvetković, I. Gutman (Eds.), Selected Topics on Applications of Graph Spectra, Math. Inst., Belgrade, 2011, pp. 113-135.

[8] I. Gutman, Hyperenergetic molecular graphs, J. Serb. Chem. Soc. 64 (1999) 199-205.

[9] I. Gutman, The energy of a graph, Ber. Math. Statist. Sekt. Forsch. Graz. 103 (1978) $1-22$.

[10] I. Gutman, S. Radenković, Hypoenergetic molecular graphs, Indian J. Chem. 46A (2007) 1733-1736.

[11] X. Li, Y. Shi, I. Gutman, Graph Energy, Springer, New York, 2012.

[12] C. D. Meyer, Matrix Analysis and Applied Linear Algebra, Soc. Industrial Appl. Math., Philadelphia, 2000.

[13] H. S. Ramane, H. B. Walikar, S. B. Rao, B. D. Acharya, P. R. Hampiholi, S. R. Jog, I. Gutman, Equienergetic graphs, Kragujevac J. Math. 26 (2004) 5-13.

[14] C. F. Van Loan, The ubiquitous Kronecker product, J. Comput. Appl. Math. 123 (2000) 85-100. 